PROCEEDINGS OF THE

AMERICAN MATHEMATICAL SOCIETY

Volume 128, Number 10, Pages 2981-2988

S 0002-9939(00)05602-1

Article electronically published on April 28, 2000

\title{
GENERICITY OF THE $K$-PROPERTY FOR A CLASS OF TRANSFORMATIONS
}

\author{
ZBIGNIEW S. KOWALSKI AND PIERRE LIARDET
}

(Communicated by Michael Handel)

\begin{abstract}
We exhibit a class of skew products over Bernoulli shifts for which the $K$-property is generic.
\end{abstract}

\section{INTRODUCTION}

Skew products over Bernoulli shifts have been considered in many papers. The particular case of isometric extensions of a two-sided Bernoulli shift with finite entropy was studied by D. Rudolph $[\mathrm{Ru}]$ who showed that such an extension is Bernoulli as soon as it is mixing. It appears that various results can be obtained if we only consider one-sided Bernoulli shifts or if we deal with non-isometric extensions. There are examples due to W. Parry $[\mathrm{Pa}]$ for which isometric extensions of a one-sided Bernoulli shift are isomorphic to the underlying shift. Meilijson $\mathrm{Me}$ investigated the case of non-isometric extensions over a Bernoulli automorphism and showed that total ergodicity of so-called power extensions implies the $K$-property. In this paper we introduce a class of skew products which are non-isometric extensions of one-sided Bernoulli shifts with finite entropy. These transformations are non-invertible, measure preserving with finite entropy and without any one-sided generator. Such transformations were first introduced in [Ko1]. Our main focus will be on the $K$-property. We exhibit dense $G_{\delta}$-sets in some compact metrizable spaces of transformations for which this latter property holds. To this end we introduce a spectral criterion which implies total ergodicity. Finally, an application to uniform distribution modulo one is given.

\section{A FAMily OF SKeW PRODUCTS}

In the sequel $a, b$ and $p$ will be fixed numbers in $] 0,1[$ with $b>p$. We introduce the set $G_{a, b}$ of continuous maps $g:[0,1] \rightarrow[0,1]$ satisfying the following properties:

(i) $g(0)=0, g(1)=1$;

(ii) $g(x) \leq x$ for any $x \in[0,1]$;

(iii) for all $(x, y) \in[0,1]^{2}$ :

$$
x \neq y \Longrightarrow a \leq \frac{g(y)-g(x)}{y-x} \leq \frac{1}{b} .
$$

Received by the editors December 1, 1998.

2000 Mathematics Subject Classification. Primary 28D05; Secondary 11K99.

The first author was supported by KBN Grant 2 P03A 03915 1998-2001. The second author was supported by MESRI. 
Notice that $G_{a, b}$ contains two particular maps, namely

$$
I(x)=\sup _{g \in G_{a, b}} g(x)
$$

which is nothing but the identity map, and

$$
\Lambda(x)=\inf _{g \in G_{a, b}} g(x)= \begin{cases}a x & \text { if } 0 \leq x \leq \frac{1-b}{1-a b} \\ \frac{1}{b} x+\left(1-\frac{1}{b}\right) & \text { otherwise. }\end{cases}
$$

Proposition 1. The set $G_{a, b}$ is a compact convex subset of the Banach space $\mathcal{C}_{\mathbb{R}}([0,1])$.

Proof. It is immediate that $G_{a, b}$ is a convex closed subset of $\mathcal{C}_{\mathbb{R}}([0,1])$ and is equicontinuous because of Property (iii). Hence $G_{a, b}$ is compact by the Ascoli Theorem.

Let $\Omega$ be the product space $\{0,1\}^{\mathbb{N}}$ endowed with the usual metrizable compact product topology and the Borel $\sigma$-algebra $\mathcal{B}_{\Omega}$. An element $w$ in $\Omega$ will be viewed as an infinite binary string $w_{0} w_{1} w_{2} \ldots$ and to every fixed finite binary string $v_{0} \ldots v_{n}$ we defined the open-closed cylinder set $C_{v_{0} \ldots v_{n}}=\left\{w \in \Omega ; w_{0} \ldots w_{n}=v_{0} \ldots v_{n}\right\}$. Given a probability $\mu$ on $\{0,1\}$ by $\mu(\{0\})=p, 0<p<1$, we associate the infinite product measure $\nu=\mu^{\infty}$ on $\Omega$ and the Bernoulli shift $B(\mu)=\left(\Omega, S, \mathcal{B}_{\Omega}, \nu\right)$ where $S$ denotes the usual one-sided shift operator. The unit interval $[0,1]$ will be endowed with its Borel $\sigma$-algebra $\mathcal{B}_{[0,1]}$ and the Lebesgue measure $\lambda$.

For every fixed homeomorphism $g$ of $[0,1]$ such that $g(0)=0$ we define the map $g_{*}:[0,1] \rightarrow[0,1]$ by the equality

$$
p g+(1-p) g_{*}=I d .
$$

If $g$ is assumed to satisfy (iii), then similar inequalities hold for $g_{*}$, namely

$$
a_{*} \leq \frac{g_{*}(y)-g_{*}(x)}{y-x} \leq \frac{1}{b_{*}}
$$

for every $x, 0 \leq x<y \leq 1$, with $a_{*}=\frac{b-p}{b(1-p)}$ and $b_{*}=\frac{1-p}{1-a p}(>p)$.

Remark 1. In general, $g_{*}$ is not necessarily one-to-one but if we assume that $g$ belongs to $G_{a, b}$, then both $g$ and $g_{*}$ are homeomorphisms of $[0,1]$.

Remark 2. For every homeomorphism $g \in G_{a, b}$ and every subinterval $J$ of $[0,1]$, the equality

$$
\lambda(J)=p \lambda(g(J))+(1-p) \lambda\left(g_{*}(J)\right)
$$

is an immediate consequence of the definition of $g_{*}$. In other words,

$$
\lambda(d x)=p \rho_{g}(d x)+(1-p) \rho_{g_{*}}(d x)
$$

where $\rho_{g}$ (resp. $\rho_{g_{*}}$ ) is the Stieljes measure whose $g$ (resp. $g_{*}$ ) is the corresponding distribution function. In particular

$$
\rho_{g}(\varphi)=\int \varphi\left(g^{-1}\right)(x) \lambda(d x)
$$

for all continuous maps $\varphi:[0,1] \rightarrow \mathbb{R}$. It follows that $\rho_{g}$ and $\rho_{g_{*}}$ are absolutely continuous with respect to $\lambda$ (and in fact equivalent to $\lambda$ ). Therefore, by the RadonNikodym Theorem, there exist non-negative functions $\gamma_{g}$ and $\gamma_{g_{*}}$ in $L^{1}(\lambda)$ such that $\rho_{g}(d x)=\gamma_{g}(x) \lambda(d x)$ and $\rho_{g_{*}}(d x)=\gamma_{g_{*}}(x) \lambda(d x)$ (equality being considered up to $\lambda$-null sets). 
For every fixed $g \in G_{a, b}$, we consider the skew product $\Sigma_{g}=\left(\Omega \times[0,1], S_{g}\right.$, $\left.\mathcal{B}_{\Omega} \otimes \mathcal{B}_{[0,1]}, \nu \otimes \lambda\right)$ over $B(\mu)$, where $S_{g}$ is the transformation defined by

$$
S_{g}(w, x)=\left(S w, T_{w_{0}}(x)\right)
$$

with $T_{0}=g^{-1}$ and $T_{1}=g_{*}^{-1}$. It follows from (2) that $S_{g}$ preserves the product measure $\sigma=\nu \otimes \lambda$. Such dynamical systems were considered by Kowalski in Ko1] and our aim is to show that the set of maps $g$ in $G_{a, b}$ such that $\Sigma_{g}$ is totally ergodic, contains a dense $G_{\delta}$ set. We first need the following weaker result:

Proposition 2. The set of $g \in G_{a, b}$ such that $\Sigma_{g}$ is totally ergodic contains a $G_{\delta}$-set with respect to the uniform topology on $G_{a, b}$.

The proof will be done in three steps and will use the spectral characterization of total ergodicity. Let $\mathcal{T}=(Y, T, \mathcal{B}, m)$ be a dynamical system and let $f \in L^{2}(m)$. By the Bochner-Herglotz Theorem, there exists a unique Borel measure $m_{f}$ (called the spectral measure of $f$ with respect to $\mathcal{T}$ ) on the torus $\mathbb{R} / \mathbb{Z}$, whose Fourier coefficients are given by

$$
\widehat{m}_{f}(k)=\left(f \circ T^{r}, f \circ T^{s}\right)
$$

where $k=r-s$. It is well known that the ergodicity of $T$ is equivalent to

$$
\forall f \in L^{2}(m):(f, 1)=0 \Longrightarrow m_{f}(\{0\})=0 .
$$

Step 1. We use the above notations with $\mathcal{T}=\Sigma_{g}$ and $g$ in $G_{a, b}$. Let $\mathcal{M}$ denote the Banach space of signed Borel measures equipped with the total variation norm. The following pair of assertions are well known: the map $f \mapsto \sigma_{f}$ from $L^{2}(\sigma)$ to $\mathcal{M}$ is continuous and, for every two functions $f_{1}, f_{2}$ in $L^{2}(\sigma)$, the spectral measure $\sigma_{f_{1}+f_{2}}$ is absolutely continuous with respect to $\sigma_{f_{1}}+\sigma_{f_{2}}$ (see $\mathrm{Qu}$ for example). Therefore, in order to prove property (4) we only have to prove it for a family $F$ of functions which generates a dense subspace of the hyperplane $L^{2}(\sigma)^{o}=\left\{f \in L^{2}(\sigma) ;(f, 1)=0\right\}$. For every fixed non-negative integer $n$, let $\psi_{n}: \Omega \rightarrow \mathcal{R}$ be the map defined by

$$
\psi_{n}(w)=\prod_{k=0}^{\infty}(-1)^{\varepsilon_{k}(n) w_{k}}\left(\frac{p \omega_{k}+(1-p)\left(1-\omega_{k}\right)}{\sqrt{p(1-p)}}\right)^{\varepsilon_{k}(n)}
$$

where $n=\sum_{k \geq 0} \varepsilon_{k}(n) 2^{k}$ is the usual expansion of $n$ in base 2 . The family $\left\{\psi_{n} ; n \in\right.$ $\mathbb{N}\}$ is an orthonormal basis in $L^{2}(\nu)$ and consequently the family

$$
F=\left\{\psi_{n} \otimes u ; n \geq 1 \& u \in L^{2}(\lambda)\right\} \cup\left\{1 \otimes u ; u \in L^{2}(\lambda) \&(u, 1)=0\right\}
$$

spans a dense subspace of $L^{2}(\sigma)^{\circ}$.

For every $f=\psi_{n} \otimes u$ in $F$ with $n \geq 1$ and for every integer $k>\log _{2} n$, a straightforward computation shows that

$$
\begin{aligned}
\widehat{\sigma}_{f}(k) & =\left(\int_{\Omega} \psi_{n} d \nu\right) \sum_{w_{0} \ldots w_{k-1}} p_{w_{0}} \ldots p_{w_{k-1}} \psi_{n}\left(w_{0}, \ldots, w_{k-1}\right) \int_{0}^{1} u\left(T_{w_{k-1}} \ldots T_{w_{0}} y\right) \overline{u(y)} \lambda(d y) \\
& =0
\end{aligned}
$$

where the sum runs over all binary strings of length $k$ and $p_{0}=p, p_{1}=1-p$. This means that $\sigma_{f}$ is absolutely continuous with respect to the Haar measure of $\mathbb{R} / \mathbb{Z}$. In particular

$$
\forall n \geq 1, \quad \sigma_{\psi_{n} \otimes u}(\{0\})=0 .
$$


Now we claim that

Lemma 1. For all $g \in G_{a, b}$ the transformation $\Sigma_{g}$ is ergodic if and only if $\sigma_{1 \otimes u}(\{0\})$ $=0$ for all $u \in L^{2}(\lambda)$ such that $(u, 1)=0$.

In fact, from (5), property (4) holds if $\sigma_{1 \otimes u}(\{0\})=0$ for every $u \in L^{2}(\lambda)$ with $(u, 1)=0$; the ergodicity of $\Sigma_{g}$ follows. The converse is obvious.

As a by-product we obtain

Proposition 3. Assume $g \in G_{a, b}$. Then $f \in L^{2}(\sigma)$ is invariant under $S_{g}$ if and only if there exists $u \in L^{2}(\lambda)$ such that $f=1 \otimes u \sigma$-a.e. and $u \circ g^{-1}=u \circ g_{*}^{-1}=u$ $\lambda$-a.e.

Proof. For every fixed $f \in L^{2}(\sigma)$ there exists a sequence $\left(u_{n}\right)_{n}$ in $L^{2}(\lambda)$ such that $f=\sum_{n=0}^{\infty} \psi_{n} \otimes u_{n}$, the series being convergent in $L^{2}$. Assume that $f$ is invariant under $S_{g}$. Then $\sigma_{f}=\|f\|_{2} \delta_{0}$ where $\delta_{0}$ stands for the Dirac probability on $\mathbb{R} / \mathbb{Z}$ at the origin. But $\sigma_{f}(\{0\})=\sigma_{1 \otimes u_{0}}(\{0\})$ from (5). Consequently $\sigma_{f}=\sigma_{1 \otimes u_{0}}$ and $u_{n}=0$ for all $n \geq 1$. Hence $f=1 \otimes u_{0}$ with $u_{0} \circ g^{-1}=u_{0} \circ g_{*}^{-1}=u_{0} \lambda$-a.e. The converse is obvious.

Step 2. Given a Borel measure $\tau$ on $\mathbb{R} / \mathbb{Z}$ we introduce the property

$$
\forall n \in \mathbb{N} \backslash\{0\}, \exists L_{n}, \max _{L_{n} \leq k<L_{n}+n}|\widehat{\tau}(k)|<\frac{1}{n} .
$$

This definition has the following application:

Lemma 2. Let $\mathcal{T}=(Y, T, \mathcal{B}, m)$ be any dynamical system and assume that for every function $f$ in $L^{2}(m)$ such that $(f, 1)=0$ the property $(E)$ holds with $\tau=m_{f}$. Then $\mathcal{T}$ is totally ergodic.

Proof. We use notations in $(E)$ with $\tau=m_{f}$. An immediate application of the Lebesgue's dominated convergence theorem gives

$$
\lim _{n \rightarrow \infty} \int_{\mathbb{R} / \mathbb{Z}}\left(\frac{1}{n} \sum_{0 \leq k<n} e^{2 i \pi\left(L_{n}+k\right) t}\right) m_{f}(d t)=m_{f}(\{0\}) .
$$

On the other hand, Property $(E)$ implies

$$
\left|\int_{\mathbb{R} / \mathbb{Z}}\left(\frac{1}{n} \sum_{0 \leq k<n} e^{2 i \pi\left(L_{n}+k\right) t}\right) m_{f}(d t)\right| \leq \frac{1}{n}
$$

and passing to the limit, we get $m_{f}(\{0\})=0$. Therefore, $T$ is ergodic. Due to the obvious fact that the Fourier coefficient at $k, k \in \mathbb{Z}$, of the spectral measure of $f$ with respect to $T^{n}$ is $m_{f}(n k)$, a similar argument shows that $T^{n}$ is also ergodic. This proves Lemma 2.

Step 3. In this last step, we assume that $g$ belongs to $G_{a, b}$ and we denote by $\sigma_{q}^{(g)}$ the spectral measure of the function $(w, y) \mapsto e^{2 i \pi q y}(q \in \mathbb{Z})$ with respect to $S_{g}$. For $n \geq 1$ given, let us introduce the subset

$$
\Gamma(q, n, L)=\left\{g \in G_{a, b} ;\left|\widehat{\sigma}_{q}^{(g)}(L+k)\right|<\frac{1}{n} \text { for } 0 \leq k<n\right\} .
$$

The next lemma will be useful to prove that the set $\Gamma(q, n, L)$ is open in $G_{a, b}$. 
Lemma 3. Let $g$ and $h$ be elements of $G_{a, b}$ and put $g_{0}=g, h_{0}=h, g_{1}=g_{*}$, $h_{1}=h_{*}$. Then, for every binary string $w_{0} \ldots w_{k-1}$ of length $k$, one has

$$
\left\|g_{w_{k-1}}^{-1} \ldots g_{w_{0}}^{-1}-h_{w_{k-1}}^{-1} \ldots h_{w_{0}}^{-1}\right\|_{\infty} \leq d\left(\frac{d^{k}-1}{d-1}\right)\|g-h\|_{\infty}
$$

where $d=\max \left\{\frac{1}{a}, \frac{b p}{b-p}, \frac{b(1-p)}{b-p}\right\}$.

Proof. We prove the assertion by induction on $k$. The case $k=1$ follows immediately from just properties (iii) or (iii $\left.{ }_{*}\right)$ and the fact that $p(g-h)+(1-p)\left(g_{*}-h_{*}\right)=0$. Now assume that inequality (6) holds for a given $k \geq 1$, so we have successively

$$
\begin{aligned}
\left\|g_{w_{k}}^{-1} \ldots g_{w_{0}}^{-1}-h_{w_{k}}^{-1} \ldots h_{w_{0}}^{-1}\right\|_{\infty} \leq & \left\|g_{w_{k}}^{-1} \circ\left(g_{w_{k-1}}^{-1} \ldots g_{w_{0}}^{-1}\right)-h_{w_{k}}^{-1} \circ\left(g_{w_{k-1}}^{-1} \ldots g_{w_{0}}^{-1}\right)\right\|_{\infty} \\
& +\left\|h_{w_{k}}^{-1} \circ\left(g_{w_{k-1}}^{-1} \ldots g_{w_{0}}^{-1}\right)-h_{w_{k}}^{-1} \circ\left(h_{w_{k-1}}^{-1} \ldots h_{w_{0}}^{-1}\right)\right\|_{\infty} \\
\leq & d\left\|g_{w_{k}}^{-1}-h_{w_{k}}^{-1}\right\|_{\infty}+d\left\|g_{w_{k-1}}^{-1} \ldots g_{w_{0}}^{-1}-h_{w_{k-1}}^{-1} \ldots h_{w_{0}}^{-1}\right\|_{\infty} \\
\leq & d\left(1+d\left(\frac{d^{k}-1}{d-1}\right)\right)\|g-h\|_{\infty} \\
& =d\left(\frac{d^{k+1}-1}{d-1}\right)\|g-h\|_{\infty} .
\end{aligned}
$$

Hence (6) holds for $k+1$, and the proof is complete.

The inequality (6) leads to

$$
\begin{aligned}
\left|\widehat{\sigma}_{q}^{(g)}(k)-\widehat{\sigma}_{q}^{(h)}(k)\right| & \leq \sum_{w_{0} \ldots w_{k-1}} \int_{0}^{1}\left|e^{2 i \pi q g_{w_{k-1}}^{-1} \ldots g_{w_{0}}^{-1}(y)}-e^{2 i \pi q h_{w_{k-1}}^{-1} \ldots h_{w_{0}}^{-1}(y)}\right| \lambda(d y) \\
& \leq 2 \pi q d\left(\frac{d^{k}-1}{d-1}\right)\|g-h\|_{\infty}
\end{aligned}
$$

and by means of a standard argument, $\Gamma(q, n, L)$ is open in $G_{a, b}$.

To complete the proof of Proposition 2, let us introduce the set

$$
\mathcal{E}=\bigcap_{q \in \mathbb{Z} \backslash\{0\}} \bigcap_{n \geq 1} \bigcap_{\ell \geq 1} \bigcup_{L \geq \ell} \Gamma(q, n, L)
$$

which is clearly a $G_{\delta}$-set. Let $\chi_{q}$ denote the exponential map $y \mapsto e^{2 i \pi q y}$ defined on $[0,1]$, choose $g \in \mathcal{E}$ and consider the family

$$
F=\left\{\psi_{n} \otimes \chi_{q} ;(n, q) \in \mathbb{N} \times \mathbb{Z} \backslash\{(0,0)\}\right\} .
$$

For every $f \in F$, the spectral measure $\sigma_{f}^{(g)}$ satisfies $(E)$. This is clear by the choice of $g$ for $f=1 \otimes \chi_{q}$. Otherwise, $f=\psi_{n} \otimes \chi_{q}$ for suitable $n$ and $q$, but in that case $\lim _{k \rightarrow \infty} \widehat{\sigma}_{f}^{(g)}(k)=0$; hence $\sigma_{f}^{(g)}$ still satisfies $(E)$. Now notice that $F \cup\{\mathbf{1}\}$ is an orthonormal basis in $L^{2}(\sigma)$ and, if $\left(f_{n}\right)$ is a sequence in $L^{2}(\sigma)$ which converges to $f$ such that all the spectral measures $\sigma_{f_{n}}^{(g)}$ satisfy property $(E)$, then $\sigma_{f}^{(g)}$ also satisfies property $(E)$. In fact, $\lim \sup _{n \rightarrow \infty} \sup _{k \in \mathbb{Z}}\left|\widehat{\sigma}_{f_{n}}^{(g)}(k)-\widehat{\sigma}_{f}^{(g)}(k)\right| \leq$ $\lim _{n \rightarrow \infty}||\left|\sigma_{f_{n}}^{(g)}-\sigma_{f}^{(g)}\right| \| \mid=0$. Here, ||$|\cdot|||$ denotes the total variation norm. Lemma 2 finishes the proof of Proposition 2.

Remark 3. There exist many functions $g$ in $G_{a, b}$ such that $S_{g}$ is not ergodic. For example, if $g$ has a fixed point in $] 0,1\left[\right.$, then $S_{g}$ cannot be ergodic. 


\section{Genericity of the $K$-Property}

Let $\Phi_{g}: L^{1}(\sigma) \rightarrow L^{1}(\sigma)$ be the Perron-Frobenius operator associated to $\Sigma_{g}$. For every $F \in L^{1}(\sigma)$, the following formula

$$
\Phi_{g}(F)(w, y)=p \gamma_{g}(y) F(0 w, g(y))+(1-p) \gamma_{g_{*}}(y) F\left(1 w, g_{*}(y)\right)
$$

is classical. We denote by $T_{g}$ the operator defined on the space $L^{1}\left([0,1], \mathcal{B}_{[0,1]}, \lambda\right)$ by

$$
T_{g}(f)=\Phi_{g}(1 \otimes f) .
$$

Notice that $T_{g}$ is doubly stochastic and the proof of the next proposition shows that the mixing property of $\Phi_{g}$ depends on that of $T_{g}$.

Proposition 4. For any $g \in G_{a, b}$, ergodicity of $\Sigma_{g}$ implies strong mixing.

Proof. Let $A: L^{1}(\lambda) \rightarrow L^{1}(\lambda)$ be the linear positive operator defined by

$$
A(h)=p h \circ g+(1-p) h \circ g_{*} .
$$

If $h \in \mathcal{C}_{\mathbb{R}}^{1}([0,1])$, then

$$
\frac{d}{d x} A h=T_{g}\left(\frac{d}{d x} h\right) \quad \lambda-\text { a.e. }
$$

Therefore, we can apply the argument of Theorem 1 in Ko2 to deduce the strong mixing property of $\Sigma_{g}$.

Using Theorem 1 in [Ko3] we obtain from Proposition 4 a similar result to the classification of D. Rudolph [Ru]:

Proposition 5. For every $g \in G_{a, b}$, the ergodicity of $\Sigma_{g}$ implies the K-property of $\Sigma_{g}$.

We are ready to study the genericity of the $K$-property from a topological point of view.

Theorem 1. Assume that $a, b, p \in] 0,1\left[, p<b\right.$. Then the set of $g$ in $G_{a, b}$ such that $\Sigma_{g}$ has the $K$-property contains a dense $G_{\delta}$-set with respect to the uniform topology.

Proof. By Propositions 1 and 5, it is enough to find a dense subset of elements in $G_{a, b}$ such that $\Sigma_{g}$ is ergodic. Let $D$ be the set of functions $g \in G_{a, b}$ such that there exists $\left.x_{0} \in\right] 0,1[$ with the following properties:

(j) The restriction of $g$ to the interval $\left[g\left(x_{0}\right), g_{*}\left(x_{0}\right)\right]$ is twice continuously differentiable;

(jj) $g^{\prime}\left(x_{0}\right)=1, g^{\prime}(x)<1$ for all $x$ in $\left[g\left(x_{0}\right), x_{0}\left[\right.\right.$ and $g^{\prime}(x)>1$ for all $\left.\left.x \in\right] x_{0}, g_{*}\left(x_{0}\right)\right]$;

(jjj) $g(x)<x$ for all $x \in] 0,1[$.

It is easily checked that $D$ is dense in $G_{a, b}$ with respect to the uniform topology. Moreover, for every $g \in D$ the skew product $\Sigma_{g}$ is ergodic. This fact is a direct consequence of the proof of Theorem 3 in [Ko1].

Open problem: In connection with the example of an exact transformation without a one-sided generator with finite entropy given in [KaKoLi], show that the exactness property is generic in $G_{a, b}$. 


\section{A family of Uniformly Distributed SEQUences}

Let $g_{0}=g$ be a homeomorphism of $[0,1]$ such that $g_{1}=g_{*}$ (the map defined by (1), section 2) is also a homeomorphism of $[0,1]$ and the sequence $n \mapsto g_{\omega_{n}}^{-1} \ldots g_{\omega_{0}}^{-1} y$ is well defined for each $\omega \in \Omega$.

Theorem 2. If $\Sigma_{g}$ is ergodic, then, for almost every point $\omega \in \Omega$, the sequence

$$
n \mapsto g_{\omega_{n}}^{-1} \ldots g_{\omega_{0}}^{-1} y
$$

is uniformly distributed in $[0,1]$ for every $y \in] 0,1[$.

Proof. Let $s(\omega, y)$ denote the sequence defined by (7). Assume that for $\omega \in \Omega$ there exist $u$ and $v$ in $] 0,1[, u<v$, such that both sequences $s(\omega, u)$ and $s(\omega, v)$ are uniformly distributed. Now, the monotonicity of $g$ and $g_{*}$ implies that, for every $t \in[0,1]$ and every $y \in[u, v]$, one has

$$
\mathbf{1}_{[0, t[}\left(s(\omega, v)_{n}\right) \leq \mathbf{1}_{[0, t]}\left(s(\omega, y)_{n}\right) \leq \mathbf{1}_{[0, t[}\left(s(\omega, u)_{n}\right) .
$$

By our assumption on $u$ and $v$ we obtain

$$
\forall t \in[0,1], \lim _{N \rightarrow \infty} \frac{1}{N} \sum_{n=0}^{N-1} \mathbf{1}_{[0, t[}\left(s(\omega, y)_{n}\right)=t .
$$

This means that the sequence $s(\omega, y)$ is uniformly distributed in $[0,1]$. But, because $S_{g}$ is ergodic, for almost all $\omega$, the set $I(\omega)$ of elements $x$ in $[0,1]$ such that $s(\omega, x)$ is uniformly distributed in $[0,1]$, has measure 1 . Therefore, the above result shows that in fact $I(\omega)=] 0,1[$.

Remark 4 . The uniformly distributed sequence $s(\omega, y)$ in Theorem 2 is not completely uniformly distributed (see $\mathrm{KuNi}$ for the definition). In fact, for every given continuous function $f:[0,1] \times[0,1] \rightarrow \mathbb{C}$, a straightforward computation shows that

$$
\begin{aligned}
\lim _{N \rightarrow \infty} \frac{1}{N} \sum_{n=0}^{N-1} f\left(s(\omega, y)_{n}, s(\omega, y)_{n+1}\right) & \\
& =p \int_{0}^{1} f\left(x, g^{-1} x\right) \lambda(d x)+(1-p) \int_{0}^{1} f\left(x, g_{*}^{-1} x\right) \lambda(d x)
\end{aligned}
$$

for all $y \in] 0,1[$ and almost all $\omega$. In particular, for such $\omega$ the sequence

$$
n \mapsto\left(s(\omega, y)_{n}, s(\omega, y)_{n+1}\right)
$$

is distributed according to a measure carried by the union of the graphs of $g^{-1}$ and $g_{*}^{-1}$.

\section{REFERENCES}

[KuNi] L. Kuipers and H. Niederreiter, Uniform Distribution of Sequences (J. Wiley, New York, ed.), 1974. MR 54:7415

[KaKoLi] B. Kamiński, Z. S. Kowalski and P. Liardet, On extremal and perfect $\sigma$-algebras for $Z^{d}$ actions on a Lebesgue space, Studia Math. 124 (2) (1997), 173-178. MR 98h:28011]

[Ko1] Z. S. Kowalski, Stationary perturbations based on Bernoulli process, Studia Math. 97 (1) (1990), 53-57. MR 92b:28021

[Ko2] Z. S. Kowalski, On mixing generalized skew products, in Ergodic theory and related topic III, Lecture Notes in Math., No 1514 (Springer-Verlag, Berlin, ed.), 1992, pp. 128-130. CMP 92:17 
[Ko3] Z. S. Kowalski, The exactness of generalized skew products, Osaka J. Math. 36 (1993), 57-61. MR 94a:28032

[Me] I. Meilijson, Mixing properties of a class of skew products, Israel J. Math. 19 (1974), 266-270. [MR 51:8374]

[Pa] W. Parry, Automorphisms of the Bernoulli endomorphism and a class of skew-products, Ergodic theory and dynamical systems 16 (1996), 519-530. MR 97h:28006

[Qu] M. Queffélec, Substitution dynamical sytems, spectral analysis, Lecture Notes in Math., No 1294 (Springer-Verlag, ed.), 1987. MR 89g:54094

$[\mathrm{Ru}] \quad$ D. Rudolph, Classifying isometric extensions of a Bernoulli shift, Journal d'Analyse Mathematique 34 (1978), 36-60. MR 80g:28020

Institute of Mathematics, Wroceaw University of Technology, Wybrzeże St. Wyspiańskiego 27, 50-370 Wroceaw, Poland

E-mail address: kowalski@im.pwr.wroc.pl

Université de Provence, CMi, 39 rue Joliot-Curie, F-13453 Marseille cedex 13, FRANCE

E-mail address: liardet@gyptis.univ-mrs.fr 